\title{
Time-Domain Techniques for Computation and Reconstruction of One-Dimensional Profiles
}

\author{
M. Rahman and R. Marklein \\ Fachgebiet Theoretische Elektrotechnik, Fachbereich Elektrotechnik/Informatik, Universität Kassel (UNIK), \\ 34109 Kassel, Germany
}

\begin{abstract}
This paper presents a time-domain technique to compute the electromagnetic fields and to reconstruct the permittivity profile within a one-dimensional medium of finite length. The medium is characterized by a permittivity as well as conductivity profile which vary only with depth. The discussed scattering problem is thus one-dimensional. The modeling tool is divided into two different schemes which are named as the forward solver and the inverse solver. The task of the forward solver is to compute the internal fields of the specimen which is performed by Greens function approach. When a known electromagnetic wave is incident normally on the media, the resulting electromagnetic field within the media can be calculated by constructing a Greens operator. This operator maps the incident field on either side of the medium to the field at an arbitrary observation point. It is nothing but a matrix of integral operators with kernels satisfying known partial differential equations. The reflection and transmission behavior of the medium is also determined from the boundary values of the Green's operator. The inverse solver is responsible for solving an inverse scattering problem by reconstructing the permittivity profile of the medium. Though it is possible to use several algorithms to solve this problem, the invariant embedding method, also known as the layer-stripping method, has been implemented here due to the advantage that it requires a finite time trace of reflection data. Here only one round trip of reflection data is used, where one round trip is defined by the time required by the pulse to propagate through the medium and back again. The inversion process begins by retrieving the reflection kernel from the reflected wave data by simply using a deconvolution technique. The rest of the task can easily be performed by applying a numerical approach to determine different profile parameters. Both the solvers have been found to have the ability to deal with different types of slabs and incident electromagnetic pulses. Slabs having continuous and discontinuous relative permittivity have already been tested successfully. The tested electromagnetic pulses are a Dirac, Gaussian and sinusoidal pulse. Due to sampling, the resolution of the system also plays a significant role in obtaining better outputs from this scheme.
\end{abstract}

Correspondence to: R. Marklein

(marklein@uni-kassel.de)

\section{Introduction}

Recently, 1-D profiling has become a point of high interest, for instance in geophysics and Non-Destructive Testing (NDT), not only due to the ease to solve the problems analytically but also due to high accuracy level. They have large importance in Time Domain Reflectometry (TDR) such as moisture meter and material analysis (Connor and Dowding, 1999; Schlaeger, 2002). The forward solver of such schemes works on a known slab and determines the electromagnetic field. On the other hand, the inverse solver takes the reflection data from the slab as an input and determines the properties of the unknown slab. The known methods to solve the forward problem are

- Finite-Difference Time-Domain (FDTD) method (Taflove and Hagness, 2000)

- Finite Integration Technique (FIT) (Marklein, 2002)

- Green's function approach (Krueger and Ochs Jr., 1989).

The Green's function approach has some advantages over the other methods. Firstly, in this method the wave equation needs not to be solved for each incident wave form. Secondly, wave field throughout the entire medium does not have to be computed. For these advantages, this method has been implemented in the forward solver. The inverse problem can be solved by

- invariant embedding (layer stripping) (Corones et al., 1983)

- downward continuation (Green's function approach) (Kristensson and Krueger, 1986, 1987).

The less amount of input data could be mentioned as main advantage of layer stripping method. Obviously the reflection data should be processed before feeding them into the inverse scheme. 


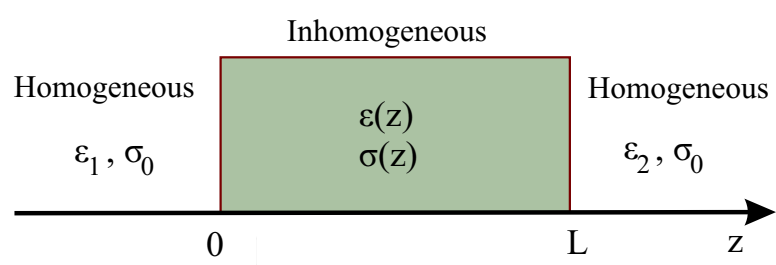

Fig. 1. Geometry of the inhomogeneous slab.

\section{Statement of the problem}

The geometry of the problem is shown in Fig. 1. An inhomogeneous slab occupies the region $0 \leq z \leq L$. The permittivity and conductivity profiles are functions of depth $z$ only.

A homogeneous, lossless medium is situated on either side of the slab. The electric field strength $E(z, t)$ within the slab can be expressed by

$$
\frac{\partial^{2}}{\partial z^{2}} E(z, t)-\frac{1}{c^{2}(z)} \frac{\partial^{2}}{\partial t^{2}} E(z, t)-b(z) \frac{\partial}{\partial t} E(z, t)=0,
$$

where

$c^{-2}(z)=\varepsilon(z) \mu_{0} \quad, \quad b(z)=\sigma(z) \mu_{0}$.

Here $\mu_{0}, \sigma(z)$ and $\varepsilon(z)$ represent the permeability of vacuum, conductivity and permittivity respectively. The phase velocity $c(z)$ is assumed to be continuous at the boundary, which means

$c(z)=c\left(0^{+}\right), \quad z \leq 0 \quad ; \quad c(z)=c\left(L^{-}\right), \quad z \geq L$.

\subsection{Normalization}

In order to facilitate the numerical computations a conversion to travel time coordinates is made. These coordinates are defined as

$$
\begin{gathered}
l=\int_{0}^{L} \frac{1}{c(z)} \mathrm{d} z \quad, \quad s(t)=t / l \\
x=x(z)=\int_{z^{\prime}=0}^{z} \frac{1}{l c\left(z^{\prime}\right)} \mathrm{d} z^{\prime} \quad, \quad u(x, s)=E(z, t),
\end{gathered}
$$

where $x$ is normalized distance and $s$ is normalized time. The slab occupies the region $0 \leq x \leq 1$ and a round trip time is described by $0<s<2$ which is equivalent to $2 l$. In fact, $l$ represents the time taken by the wave front to travel through the slab once. So the wave equation will be transformed into

$$
\begin{aligned}
\frac{\partial^{2}}{\partial x^{2}} u(x, s)-\frac{\partial^{2}}{\partial s^{2}} u(x, s) & +A(x) \frac{\partial}{\partial x} u(x, s) \\
& +B(x) \frac{\partial}{\partial s} u(x, s)=0,
\end{aligned}
$$

where

$$
\begin{aligned}
& A(x)=-\frac{\mathrm{d}}{\mathrm{d} x} \ln c[z(x)] \\
& B(x)=-l b[z(x)] c^{2}[z(x)] .
\end{aligned}
$$

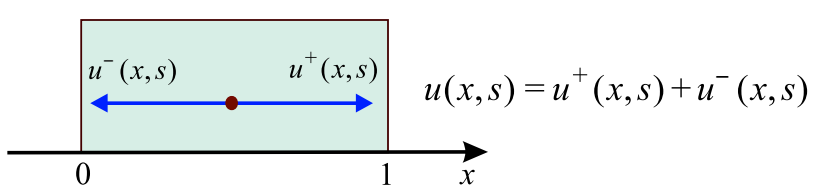

Fig. 2. Wave splitting.

\subsection{Wave splitting}

The wave travelling in the non-homogeneous slab can be splitted into left- and right-going parts. In Fig. $2, u^{+}(x, s)$ is the right going wave and $u^{-}(x, s)$ is the left going wave. For all time $s$, the initial and boundary conditions are

$$
\begin{array}{rll}
u^{+}(x, 0)=u^{-}(x, 0)=0 & ; & 0<x<1 \\
u^{+}(0, s)=f(s) \quad, \quad u^{-}(1, s)=0 & ; & s>0 .
\end{array}
$$

An incident wave is impinged only from the left side which is denoted by $f(s)$.

\section{Forward solver: introduction to the Green's kernel}

The known parameters are incident pulse, slab length $L, \varepsilon_{\mathrm{r}}$ and $\sigma$ profile. The goal is to determine the internal fields. A matrix operator $[\mathbf{G}](x)$ can be defined to map the incident waves $u^{+}(0, s), u^{-}(1, s)$ to internal fields $u^{+}(x, s), u^{-}(x, s)$

$$
\left\{\begin{array}{l}
u^{+}(x, s) \\
u^{-}(x, s)
\end{array}\right\}=\underbrace{\left[\begin{array}{ll}
G_{11}(x) & G_{12}(x) \\
G_{21}(x) & G_{22}(x)
\end{array}\right]}_{=[\mathbf{G}](x)}\left\{\begin{array}{l}
u^{+}(0, s) \\
u^{-}(1, s)
\end{array}\right\} .
$$

Considering an incident wave only from left side, we find

$$
\left.\begin{array}{l}
u^{+}(x, s)=G_{11}(x) * f(s) \\
u^{-}(x, s)=G_{21}(x) * f(s)
\end{array}\right\} ; \text { as } u^{+}(0, s)=f(s),
$$

where "*" denotes convolution. $G_{11}, G_{21}$ are related to the forward and backward travelling wave, respectively, when the incident wave is applied from the left side of the slab. $G_{12}, G_{22}$ are not considered here as they are only applicable for an incident wave coming from the right side of the slab. Now by applying Duhamel's integral,

$$
\begin{aligned}
u^{+}(x, s)= & t^{+}(0, x)\{f(s-x) \\
& \left.+\int_{0}^{s-x} f\left(s^{\prime}\right) G_{11}\left(x, s-s^{\prime}\right) \mathrm{d} s^{\prime}\right\} \\
u^{-}(x, s)= & \frac{1}{t^{-}(0, x)} \int_{0}^{s-x} f\left(s^{\prime}\right) G_{21}\left(x, s-s^{\prime}\right) \mathrm{d} s^{\prime},
\end{aligned}
$$

where

$$
t^{ \pm}\left(x_{1}, x_{2}\right)=\exp \left\{ \pm \frac{1}{2} \int_{x_{1}}^{x_{2}}\left[A\left(x^{\prime}\right] \mp B\left(x^{\prime}\right)\right] \mathrm{d} x^{\prime}\right\} .
$$




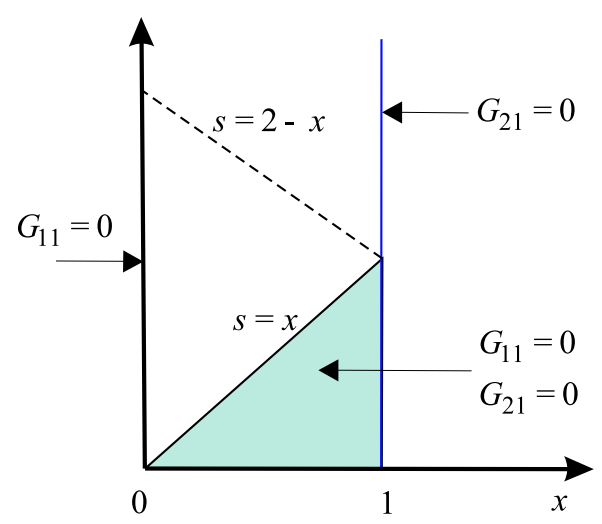

Fig. 3. Conditions of the Green's kernel.

\subsection{Equations of the Green's kernel}

The fields $u^{+}(x, s)$ and $u^{-}(x, s)$ satisfy the following equation

$$
\frac{\partial}{\partial x}\left\{\begin{array}{l}
u^{+}(x, s) \\
u^{-}(x, s)
\end{array}\right\}=\left[\begin{array}{ll}
\alpha(x) & \beta(x) \\
\gamma(x) & \delta(x)
\end{array}\right]\left\{\begin{array}{l}
u^{+}(x, s) \\
u^{-}(x, s)
\end{array}\right\},
$$

where $\alpha(x), \beta(x), \gamma(x)$, and $\delta(x)$ are the functions of $A(x)$, $B(x)$ and incorporate time derivative $\frac{\partial}{\partial s}$. From Eqs. (11) and (10), it can be summarized that

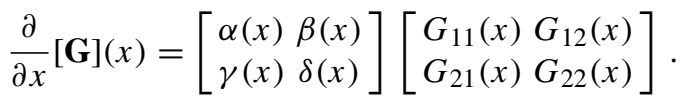

Again using Eq. (7), it can be shown that

$$
\begin{aligned}
\frac{\partial}{\partial x}\left[G_{11}(x) f\right](s)= & \alpha(x)\left[G_{11}(x) f\right](s) \\
& +\beta(x)\left[G_{21}(x) f\right](s) .
\end{aligned}
$$

Substituting $\left[G_{11}(x) f\right](s)$ by the right side of the Eq. (13), a simple expression of $G_{11}$ can be obtained, as follows

$$
\begin{aligned}
& \frac{\partial}{\partial x} G_{11}(x, s)+\frac{\partial}{\partial s} G_{11}(x, s) \\
& =\frac{1}{2}[A(x)+B(x)] \exp \left\{-\int_{0}^{x} B\left(x^{\prime}\right) \mathrm{d} x^{\prime}\right\} G_{21}(x, s) .
\end{aligned}
$$

Similarly using Eq. (14), $G_{21}$ can be defined as

$$
\begin{aligned}
& \frac{\partial}{\partial x} G_{21}(x, s)+\frac{\partial}{\partial s} G_{21}(x, s) \\
& =\frac{1}{2}[A(x)-B(x)] \exp \left\{\int_{0}^{x} B\left(x^{\prime}\right) \mathrm{d} x^{\prime}\right\} G_{11}(x, s) .
\end{aligned}
$$

\subsection{Initial, boundary, and jump conditions}

$G_{11}$ is continuous everywhere except on $s=x$ (see Fig. 3). On this line the kernels can be expressed as

$$
\begin{aligned}
& G_{11}\left(x, x^{+}\right)=-\frac{1}{8} \int_{0}^{x}\left[A^{2}\left(x^{\prime}\right)-B^{2}\left(x^{\prime}\right)\right] \mathrm{d} x^{\prime} \\
& G_{21}\left(x, x^{+}\right)=-\frac{1}{4}[A(x)-B(x)] \exp \left\{\int_{0}^{x} B\left(x^{\prime}\right) \mathrm{d} x^{\prime}\right\} .
\end{aligned}
$$

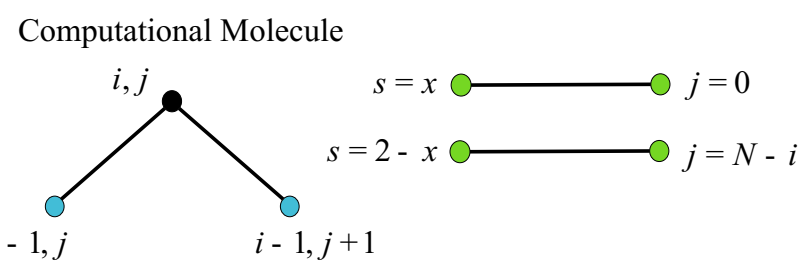

Fig. 4. Computational molecule and variable transformation.

Again on the line $s=2-x$,

$G_{21}\left(x,(2-x)^{+}\right)-G_{21}\left(x,(2-x)^{-}\right)$

$=\frac{1}{4}[A(1)-B(1)] \exp \left\{\int_{0}^{1} B\left(x^{\prime}\right) \mathrm{d} x^{\prime}\right\}$.

The initial and boundary conditions can be defined as

$G_{11}(x, s)=G_{21}(x, s)=0 ; s<x$

$G_{11}(0, s)=G_{21}(1, s)=0 ; s>0$.

\subsection{Numerical implementation of the forward solver}

The Green's kernels explained by Eqs. (19) and (20) can also be implemented numerically. By fixing a constant $\Delta$, the first equation can be integrated from $(x-\Delta, s-\Delta)$ to $(x, s)$ along the characteristics $s-x=$ const. and the second one from $(x, s)$ to $(x+\Delta, s-\Delta)$ along the characteristics $s+x=$ const. with approximating the right-hand side by trapezoidal rule. So the equations will become

$$
\begin{array}{r}
G_{11}(x, s)-G_{11}(x-\Delta, s-\Delta)=a(x) G_{21}(x, s) \\
+a(x-\Delta) G_{21}(x-\Delta, s-\Delta)+O\left(\Delta^{3}\right) \\
G_{21}(x+\Delta, s-\Delta)-G_{21}(x, s)=b(x) G_{11}(x, s) \\
+b(x+\Delta) G_{11}(x+\Delta, s-\Delta)+O\left(\Delta^{3}\right),
\end{array}
$$

where

$$
\begin{aligned}
& a(x)=\frac{1}{4} \Delta[A(x)+B(x)] \exp \left(-\int_{x^{\prime}=0}^{x} B\left(x^{\prime}\right) \mathrm{d} x^{\prime}\right) \\
& b(x)=\frac{1}{4} \Delta[A(x)-B(x)] \exp \left(\int_{x^{\prime}=0}^{x} B\left(x^{\prime}\right) \mathrm{d} x^{\prime}\right) .
\end{aligned}
$$

Let us now introduce a grid of points as shown in Fig. 4: $\left(x_{i}, s_{i+2 j}\right)=(i \Delta,(i+2 j) \Delta), i=0, \ldots, N ; j=0, \ldots$ Here $N$ represents the total number of grid points. Considering $\Delta=1 / N$, it can be stated that

$G_{11}^{i, j}=G_{11}(i \Delta,(i+2 j) \Delta), \quad a^{i}=a(i \Delta)$

$G_{21}^{i, j}=G_{21}(i \Delta,(i+2 j) \Delta), \quad b^{i}=b(i \Delta)$.

Using these approximations, Eqs. (26) and (27) can be rewritten as

$$
\begin{gathered}
G_{11}^{i, j}=d^{i}\left[G_{11}^{i-1, j}+a^{i-1} G_{21}^{i-1, j}-a^{i} b^{i+1} G_{11}^{i+1, j-1}\right. \\
\left.+a^{i} G_{21}^{i+1, j-1}\right] \\
G_{21}^{i, j}=d^{i}\left[-b^{i} G_{11}^{i-1, j}-a^{i-1} b^{i} G_{21}^{i-1, j}-b^{i+1} G_{11}^{i+1, j-1}\right. \\
\left.+G_{21}^{i+1, j-1}\right]
\end{gathered}
$$



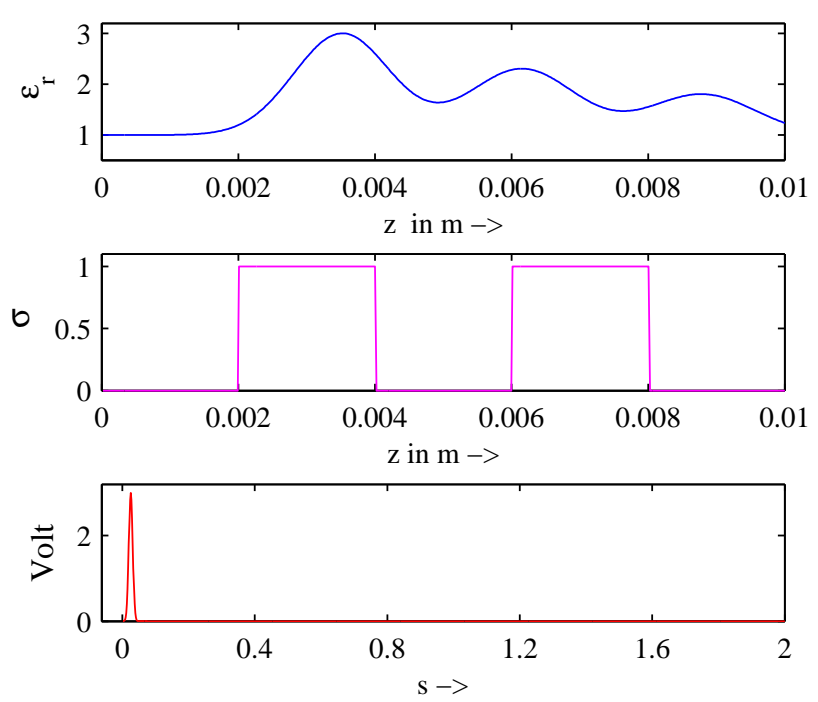

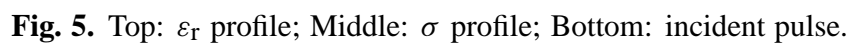
Slab length $L=1 \mathrm{~cm}$, number of cells in both $z$ and $x$ direction is 512 , grid size $\Delta=1.95 \times 10^{-5} \mathrm{~m}$ and $\Delta t=85 \mathrm{ps}$.

where

$d^{i}=\left[1+a^{i} b^{i}\right]^{-1}=\left[1+\frac{1}{16} \Delta^{2}\left(A^{2}(i \Delta)-B^{2}(i \Delta)\right)\right]^{-1}$

and $i=1,2, \ldots, N-1 ; j=1,2, \ldots$ The initial condition represented by $s=x$, i.e., $j=0$, can be determined by Eqs. (21) and (22). The boundary conditions are

$G_{11}^{0, j}=0, \quad G_{21}^{N, j}=0 ; \quad j=0,1,2, \ldots$

To incorporate the discontinuity of $G_{21}$ on the line $s=2-x$, i.e. $j=N-i, G_{21}^{i, N-i}$ below the line can be computed by Eq. (33). Above this line, $\left(G_{21}^{i, N-i}\right)^{+}$can be determined by

$$
\begin{aligned}
\left(G_{21}^{i, N-i}\right)^{+} & =\left(G_{21}^{i, N-i}\right)^{-} \\
+ & \frac{1}{4}[A(1)-B(1)] \exp \left\{\int_{0}^{1} B(x) \mathrm{d} x\right\} .
\end{aligned}
$$

This value is used in place of $G_{21}^{i, N-i}$ determined by Eq. (33) while computing $G_{11}^{i, N-i+1}$ and $G_{21}^{i, N-i+1}$.

\subsection{Software implementation of the forward solver}

This scheme has already been tested to simulate sinusoidal, square and multiple Gaussian shaped $\varepsilon_{\mathrm{r}}$ profiles. Considered electromagnetic pulses are: Dirac pulse, Gaussian pulse, five pulses of sinusoidal wave, raised cosine and step function. The results have been tested in both lossless and lossy condition. A multiple Gaussian shaped slab with incident Gaussian pulse has been simulated here (see Figs. 5 and 6).

\section{Inverse solver: layer stripping}

The necessary data to start inversion are the length of the slab $L$, incident pulse (as reference), relative permittivity $\varepsilon_{1}$ of the

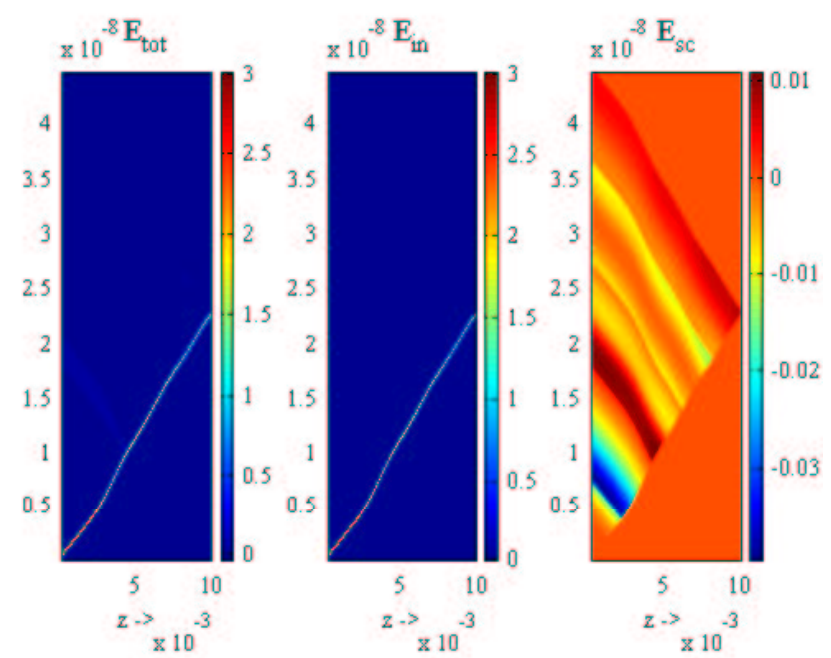

Fig. 6. Left: Total field $E_{\text {total }}$; Middle: Incident field $E^{+}$; Right: Reflected field $E^{-}$.

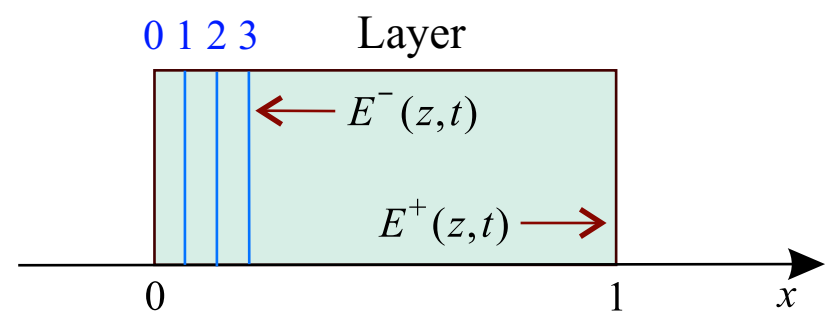

Fig. 7. Wave splitting and layer approach.

first medium (surrounding medium) and measured reflection data. The desired goal is to determine the unknown relative permittivity $\varepsilon_{\mathrm{r}}$ profile of the slab. In the inversion scheme, the slab is assumed to be lossless, so the conductivity $\sigma$ becomes zero. From the wave splitting phenomenon, it can be shown that

$E(z, t)=E^{+}(z, t)+E^{-}(z, t)$.

The backward travelling wave $E^{-}(z, t)$ can be associated with reflection kernel $R^{+}(z, t)$ in the following way

$E^{-}(z, t)=E^{+}(z, t) * R^{+}(z, t)$.

The reflection data obtained from measurement can be mentioned as $E^{-}(0, t)$ at the first layer (see Fig. 7). To find the reflection kernel $R^{+}(0, t)$ at this layer, we have to perform deconvolution. As a result

$R^{+}(0, t)=\operatorname{decon}\left\{E^{-}(0, t), f(t)\right\}$ as $f(t)=E^{+}(0, t)$.

\subsection{Input data processing}

As deconvolution is much easier to perform in frequency domain, the time domain parameters $f(t)$ and $E^{-}(0, t)$ will be transformed into frequency domain parameters $F(\omega)$ and $E^{-}(\omega)$ at first by using Fast Fourier Transform (FFT). Then 
in frequency domain it can be written as

$E^{-}(\omega)=R^{+}(\omega) F(\omega) \Rightarrow R^{+}(\omega)=E^{-}(\omega) / F(\omega)$

The obtained data should be filtered in order to avoid zeros which might occur at the deconvolution step. A Hanning window is used in this scheme as a filter. At the end, $R^{+}(\omega)$ is converted into $R^{+}(0, t)$ by Inverse Fast Fourier Transform (IFFT). Then the reflection data from the first layer will be used to initiate the reconstruction process.

\subsection{Reflection kernel}

Equation (38) can be rewritten as

$E^{-}(z, t)=\int_{-\infty}^{t} R^{+}\left(z, t-t^{\prime}\right) E^{+}\left(z, t^{\prime}\right) \mathrm{d} t^{\prime}$.

Solving this equation for $R^{+}(z, t)$,

$$
\begin{aligned}
\frac{\partial}{\partial z} R^{+}(z, t) & -\frac{2}{c(z)} \frac{\partial}{\partial t} R^{+}(z, t) \\
& =\frac{c^{\prime}(z)}{2 c(z)} \int_{0}^{t} R^{+}\left(z, t-t^{\prime}\right) R^{+}\left(z, t^{\prime}\right) \mathrm{d} t^{\prime} \\
R^{+}(z, 0) & =\frac{1}{4} c^{\prime}(z) \\
R^{+}(L, t) & =0 .
\end{aligned}
$$

Before entering the reconstruction step, normalization will be performed according to the principle explained in Sect. 2.1. So the reflection kernel $R^{+}(z, t)$ will be transformed into $R^{+}(x, s)$ according to the relationship $R^{+}(x, s)=l R^{+}(z, t)$. Equations (42)-(44) will become

$$
\begin{aligned}
\frac{\partial}{\partial x} R^{+}(x, s) & -2 \frac{\partial}{\partial s} R^{+}(x, s) \\
& =-\frac{A(x)}{2} \int_{0}^{s} R^{+}\left(x, s-s^{\prime}\right) R^{+}\left(x, s^{\prime}\right) \mathrm{d} s^{\prime} \\
R^{+}(x, 0) & =-\frac{1}{4} A(x) \\
R^{+}(1, s) & =0
\end{aligned}
$$

\subsection{Reconstruction basics}

The goal of this part is to determine the mapping parameter $z(x)$ and the permittivity profile $\varepsilon[z(x)]$. From Eq. (7)

$$
\begin{aligned}
& A(x)=-\frac{\mathrm{d}}{\mathrm{d} x} \ln c[z(x)] \\
\Rightarrow & \ln c[z(x)]=-\int_{0}^{x} A\left(x^{\prime}\right) \mathrm{d} x^{\prime}+\text { const. } \\
\Rightarrow & c[z(x)]=k \exp \left\{-\int_{0}^{x} A\left(x^{\prime}\right) \mathrm{d} x^{\prime}\right\} .
\end{aligned}
$$

The constant $k$ represents $c(0)$, the phase velocity of the first layer, i.e at $x=0$. Now from normalization principle,

$$
\begin{gathered}
x(z)=\int_{0}^{z} \frac{1}{l c\left(z^{\prime}\right)} \mathrm{d} z^{\prime} \Rightarrow \frac{\mathrm{d}}{\mathrm{d} z} x(z)=-\frac{1}{l c(z)} \\
\Rightarrow c(z(x))=-\frac{1}{l} \frac{\mathrm{d}}{\mathrm{d} x} z(x) .
\end{gathered}
$$

From Eqs. (50) and (52) it can be summed up that

$$
z(x)=c(0) l \int_{0}^{x}\left[\exp \left\{-\int_{0}^{x^{\prime}} A\left(x^{\prime \prime}\right) \mathrm{d} x^{\prime \prime}\right\} \mathrm{d} x^{\prime}\right] \text {. }
$$

Replacing $c(z)$ by $1 / \sqrt{\mu \varepsilon(z)}$, Eq. (50) can be rewritten as

$$
\begin{aligned}
& \frac{1}{\mu \varepsilon(z)}=\frac{1}{\mu \varepsilon(0)} \exp \left\{-2 \int_{0}^{x} A\left(x^{\prime}\right) \mathrm{d} x^{\prime}\right\} \\
& \Rightarrow \varepsilon[z(x)]=\varepsilon_{1} \exp \left\{2 \int_{0}^{x} A\left(x^{\prime}\right) \mathrm{d} x^{\prime}\right\} ; 0<x<1,
\end{aligned}
$$

where $\varepsilon_{1}=\varepsilon(0)$ represents the permittivity of the first layer or the first medium. Eqs. (53) and (55) are the basic equations for reconstruction.

\subsection{Numerical implementation of the inverse solver}

The embedding equation (Eq. 45) can be rewritten as

$$
\begin{aligned}
& \frac{\partial}{\partial x} R^{+}(x, s-2 x)= \\
& -\frac{1}{2} A(x) \int_{0}^{s-2 x} R^{+}\left(x, s-2 x-s^{\prime}\right) R^{+}\left(x, s^{\prime}\right) \mathrm{d} s^{\prime} .
\end{aligned}
$$

Let us fix a constant $\Delta(=\Delta x=\Delta s)$. Integrating this equation from $x-\Delta$ to $x$ and keeping time at $s+2 x$,

$$
\begin{aligned}
& R^{+}(x, s)-R^{+}(x-\Delta, s+2 \Delta)= \\
& -\frac{1}{2} \int_{x-\Delta}^{x} A\left(x^{\prime}\right)\left(R^{+} * R^{+}\right)\left(x^{\prime}, s+2\left(x-x^{\prime}\right)\right) \mathrm{d} x^{\prime},
\end{aligned}
$$

where

$$
\left(R^{+} * R^{+}\right)(x, s)=\int_{0}^{s} R^{+}\left(x, s-s^{\prime}\right) R^{+}\left(x, s^{\prime}\right) \mathrm{d} s^{\prime} .
$$

Let us introduce a uniform grid of points $\left(x_{i}, s_{j}\right)$ :

$x_{i}=i \Delta, \quad i=0,1, \ldots, N$

$s_{j}=2 j \Delta, \quad j=0,1, \ldots, N-i$.

Considering $\Delta=1 / N$, where $N$ is the number of grid points,

$R_{i, j}=R\left(x_{i}, s_{j}\right) \quad ; \quad A_{i}=A\left(x_{i}\right)$.

Using this grid point, Eq. (56) will be transformed into

$$
\begin{aligned}
& R_{i, j}^{+}=\left[R_{i-1, j+1}^{+}-\frac{\Delta^{2}}{2}\left\{A_{i} \sum_{k=1}^{j-1} R_{i, j-k}^{+} R_{i, k}^{+}\right.\right. \\
& \left.\left.+A_{i-1} \sum_{k=1}^{j+1} R_{i-1, j+1-k}^{+} R_{i-1, k}^{+}\right\}\right]\left(1-\frac{\Delta^{2}}{8} A_{i}^{2}\right)^{-1} .
\end{aligned}
$$

Again from Eq. (46),

$A_{i}=-4 R_{i-1,1}^{+}\left\{1+\frac{\Delta^{2}}{8} A_{i-1}^{2}\right\}$.

The error made in Eqs. (62) and (63) is of order $O\left(\Delta^{3}\right)$. The initialization of the algorithm is made by assigning,

$R_{0, j}^{+}=R^{+}(0,2 j \Delta)=l R^{+}(2 j l \Delta) ; \quad j=0,1, \ldots, N$. 


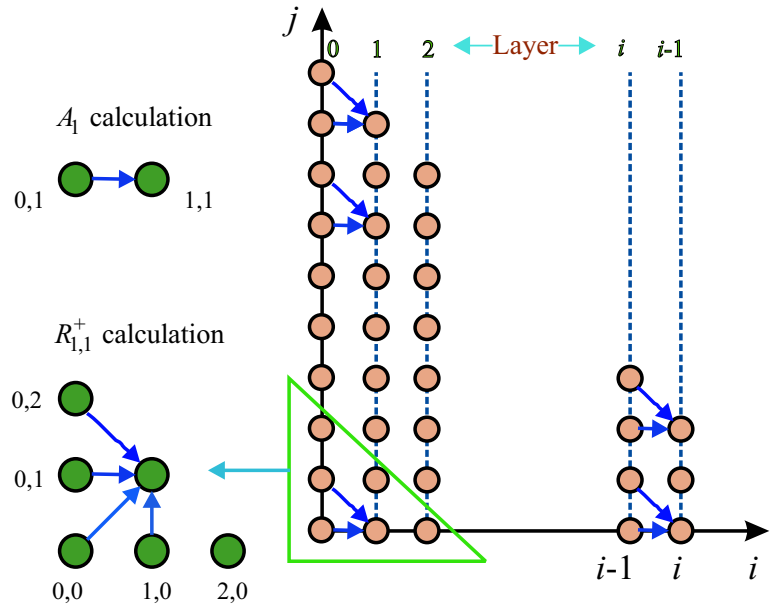

Fig. 8. Numerical algorithm of inversion scheme.
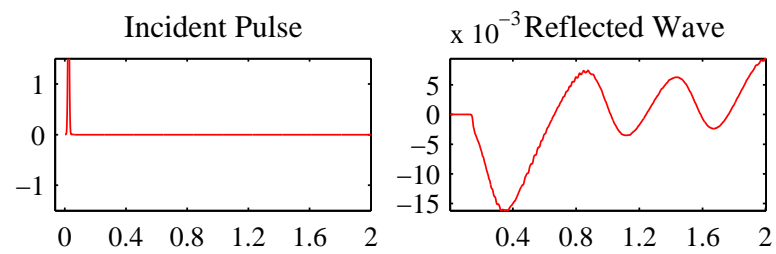

After FFT Incident (Real)
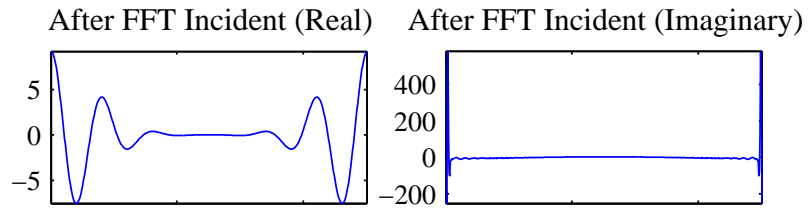

After FFT Reflected (Real) After FFT Reflected (Imaginary)
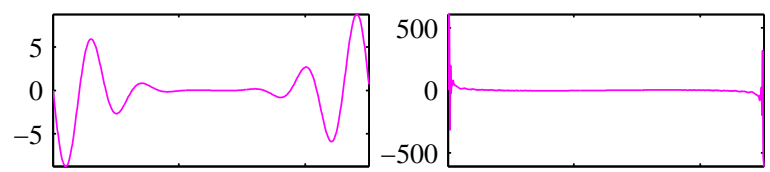

Fig. 9. Incident wave, reflected wave and FFT results. $L=1 \mathrm{~cm}$, $N_{z}=N_{x}=512, \Delta=1.95 \times 10^{-5} \mathrm{~m}, \Delta t=85 \mathrm{ps}$.

The reconstruction is done in two steps as shown in in Fig. 8. At first, $A\left(x_{i}\right)$ is calculated from $(i-1)$-th grid. Secondly $R_{i, j}^{+}$is calculated from current time step data of $(i-1)$-th grid, old time step data of $i$-th grid and next time step data of $i-1$-th grid.

\subsection{Software implementation of the inverse solver}

The inversion scheme has already been tested to reconstruct sinusoidal shaped, square shaped and multiple Gaussian shaped relative permittivity profiles using the reflection data from Dirac pulse, Gaussian pulse, five pulses of sinusoidal wave, raised cosine and step function in both noisy and noise free environment. A multiple Gaussian shaped $\varepsilon_{\mathrm{r}}$ profile has been reconstructed here as an example simulation (see Figs. 9 and 10).

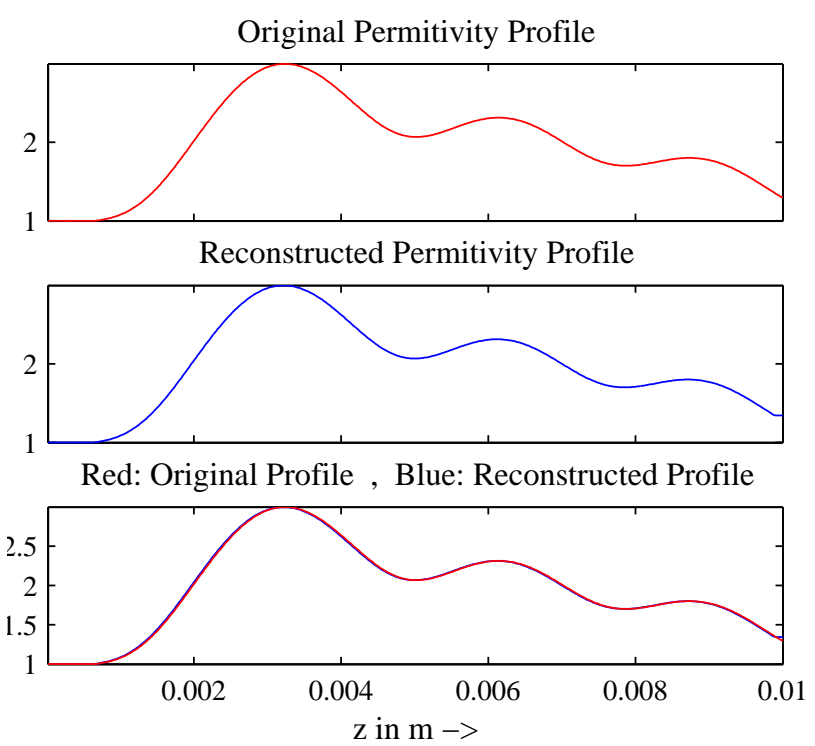

Fig. 10. Top: Original profile; Middle: Reconstructed profile; Bottom: Superposition of original and reconstructed profile.

\section{Conclusions}

The inverse solver presented in this paper shows almost accurate reconstruction. Better output can be generated by using higher resolutions. The tested resolutions are 128, 256, 512, 1024 and 2048. Besides all the combinations of relative permittivity and incident pulses have already been checked with synthetic and measured data. From the recent observations it has been proved that the inverse solver shows satisfactory result under noisy environment, too. Further modifications of the inversion algorithm are still going on. The next step is to reconstruct the permittivity and conductivity profiles from reflection and transmission data in lossy case.

\section{References}

Connor, K. M. and Dowding, C. H.: GeoMeasurements by Pulsing TDR Cables and Probes, CRC Press, Boca Raton, USA, 1999.

Corones, J. P., Davison, M. E. and Krueger, R. J.: Wave splittings, invariant embedding and inverse scattering, Inverse Optics, Proc. SPIE, 413, 102-106, 1983.

Kristensson, G. and Krueger, R. J.: Direct and inverse scattering in the time domain for a dissipative wave equation. Part 1 and Part 2, Journal of Mathematical Physics, 27, 1667-1693, 1986.

Kristensson, G. and Krueger, R. J.: Direct and inverse scattering in the time domain for a dissipative wave equation. Part 3: Scattering operators in presence of phase velocity mismatch, Journal of Mathematical Physics, 28, 360-370, 1987.

Krueger, R. J. and Ochs Jr., R. L.: A Green's function approach to the determination of internal fields, Applied Mathematical Sciences, 11, 525-543, 1989.

Marklein, R.: The Finite Integration Technique as a General Tool to Compute Acoustic, Electromagnetic, Elastodynamic, and Coupled Wave Fields, in: Review of Radio Science: 1999-2002 
URSI, edited by: Stone, W. R., IEEE Press, Piscataway, 201244, 2002.

Schlaeger, S.: Inveriosn of TDR Measurements to Reconstruct Spatially Distributed Geophysical Ground Parameter, Ph.D. Thesis (in German), Karlsruhe, Germany, 2002.
Taflove, A. and Hagness, S. C.: Computational Electrodynamics: The Finite-Difference Time-Domain Method, 2nd ed., Artech House, Boston, 2000. 